\title{
ANALYSIS OF PRESSURE LOSS COMPONENTS IN AN INDUSTRIAL EXTRACT DUCT SYSTEM
}

\author{
JOHN IRISOWENGIBIA SODIKI ${ }^{1^{*}}$ \\ ${ }^{I}$ Department of Mechanical Engineering Rivers State University of Science and Technology, \\ P.M.B.5080, Port Harcourt, Nigeria
}

\begin{abstract}
The frictional head loss and the loss through fittings are computed for branch duct runs of an industrial extract ventilation system. Results show increases of both of the aforementioned loss components with increase in duct length. Furthermore, the fraction of the total loss due to fittings decreases from 0.60 to 0.45 , with a corresponding increase of the fraction due to friction (from 0.40 to 0.55 ).Representative fractions of head loss components, obtained in the manner of this study, are shown to facilitate loss estimates and extract fan selection.
\end{abstract}

Keywords: index duct runs, industrial ventilation, head loss components

\section{INTRODUCTION}

The total head loss in an index duct run of an extract ventilation system comprises the losses through duct friction, fittings (such as elbows, tees and enlargements) and duct accessories (such as grilles, weather louvers and sound attenuators). The ventilating fan static pressure (which needs to be carefully estimated for proper fan selection) should exceed the sum of this total loss and the terminal pressure at the fan discharge. Usually, the estimation of the frictional loss and that due to fittings requires a greater effort than determining the other components of the fan pressure; the latter components being easily determined from the equipment manufacturer's technical specifications. To aid this effort, an earlier study had been carried out where a relationship between the total frictional loss and total loss due to fittings in composite index runs had been obtained, for varying duct complexities in an extract ventilation system serving groups of toilet rooms [1]. Thus, a representative fraction due to all installed fittings in an index duct run may simply be added to the frictional loss to obtain a total and, thereby, serve in facilitating the fan selection procedure.

The present study is a case of an industrial ventilation system serving a canteen, kitchen and ablution spaces. The variation of the loss components, the total loss, and the fraction of the loss due to duct fittings with varying lengths of index run in the duct configuration are studied.

\section{VENTILATION SYSTEM DESCRIPTION}

The system is shown in the floor plan of Figure 1 and the isometric sketch of Figure 2. The following duct runs are utilized to study the variation of component and total pressure losses with length of duct run:
a. $\quad 0,1,2,--, 12$
b. $\quad 0,1,2,--, 10,13,14$

\footnotetext{
${ }^{*}$ Corresponding author, email: jisodiki partners@yahoo.com

(C) 2016 Alma Mater Publishing House
} 
c. $\quad 0,1,2,--, 9,15,16$

d. $\quad 0,1,2,--, 7,17,18$

e. $\quad 0,1,2,--, 5,19$

f. $\quad 0,1,2,3,20,--, 24$

g. $\quad 0,1,2,3,20,25$

h. $\quad 0,1,26,---, 3$

i. $\quad 0,1,26,27,32$

In Figure 2 the duct runs are labelled by boxes such that the air quantity (in $\mathrm{m}^{3} / \mathrm{min}$ ) is on the top and the duct length (in $\mathrm{m}$ ) is on the bottom. For all the duct runs, the following system parameters are adopted in order to provide a common basis for their analyses:

a. Recommended ventilation rates of $0.34 \mathrm{~m}^{3} / \mathrm{min}$ per person, $0.60 \mathrm{~m}^{3} / \mathrm{min} / \mathrm{m}^{2}$ of floor area and 1.2 $\mathrm{m}^{3} / \mathrm{min} / \mathrm{m}^{2}$ of floor area, respectively, for cafeteria, toilets and kitchens [2] are utilized.

b. On account of reducing sound levels in the ductwork, flow velocities between $4.5 \mathrm{~m} / \mathrm{s}$ and $8.0 \mathrm{~m} / \mathrm{s}$ are recommended in ventilation ducts [3]. An average value of $6.25 \mathrm{~m} / \mathrm{s}$ is utilized.

c. Pressure losses through duct accessories, such as intake grilles, weather louvers and sound attenuation, whose values are usually provided by their specialist manufacturers, are not included in the analysis. However, such loss values should normally be added to obtain a total head loss.

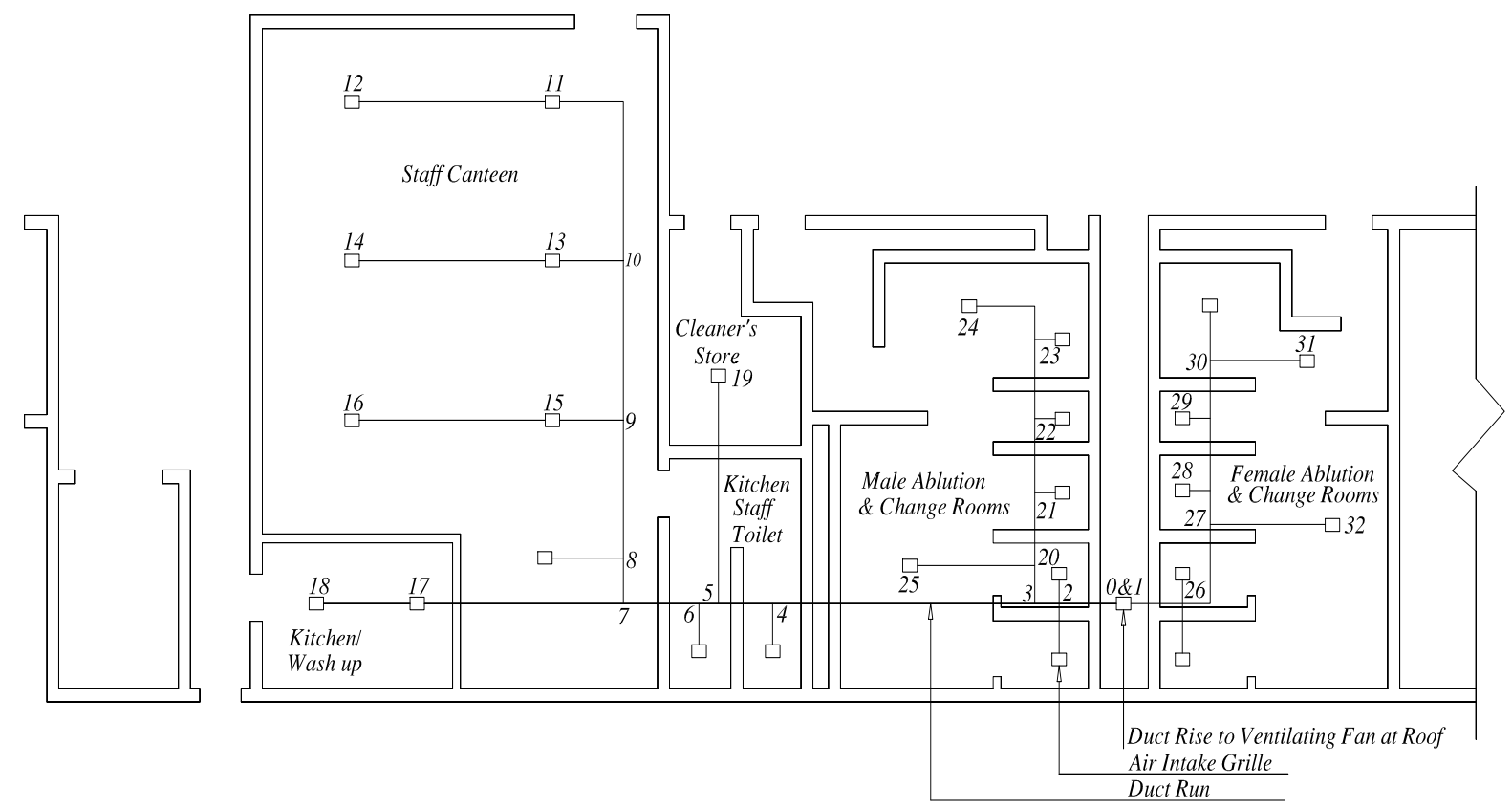

Fig. 1. Floor plan of ventilation ductwork.

\section{METHODS ADOPTED}

The system parameters are calculated by the following methods.

\subsection{Duct Sizing}

By utilizing the flow velocity of $6.25 \mathrm{~m} / \mathrm{s}$ and the respective air quantities $\mathrm{q}$ (in $\mathrm{m}^{3} / \mathrm{s}$ ) in each duct section, the round duct size for each section is obtained as:

$$
d=\sqrt{\frac{4 q}{6.25 \pi}}=0.4513 q^{1 / 2}[m]
$$

\subsection{Calculation of Frictional Losses}

For composite duct runs, the total friction head loss $h_{\text {friction }}$ as obtained as [4]. 


$$
h_{\text {friction }}=0.3304 \sum_{i=1}^{i=n} \frac{f_{i} l_{i} q_{1}^{2}}{d_{i}^{5}}[m]
$$

where $f$ is the duct section friction factor, $l$ is the section length (in m); $i$ denotes the $i^{\text {th }}$ duct section and $n$ is the number of sections in the composite run; $q$ and $d$ are as defined earlier; $f$ is a function of the flow Reynolds number Re given as:

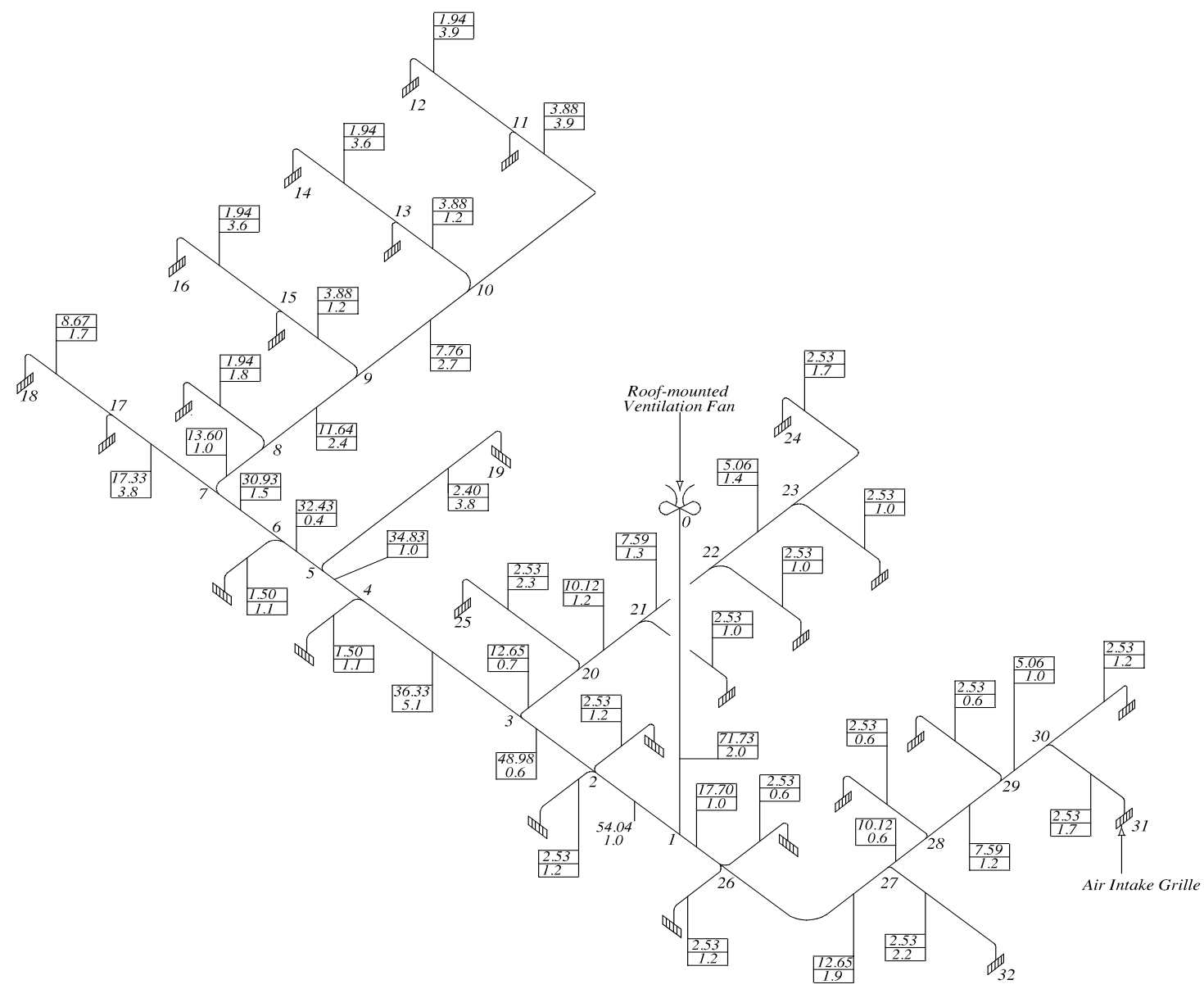

Fig. 2. Isometric sketch of ventilation ductwork.

$$
\operatorname{Re}=\frac{\rho v d}{\mu}
$$

where $\rho$ is the air density (taken as $1.2 \mathrm{~kg} / \mathrm{m}^{3}$ ), $v$ is the flow velocity, and $\mu$ is the air dynamic viscosity (taken as $1.8 \times 10^{-5} \mathrm{~kg} / \mathrm{ms}$ ). Expressing $v$ in terms of flow rate and duct diameter, Re may then be expressed as:

$$
\operatorname{Re}=8.515 \times 10^{4} q / d
$$

For the determination of $\mathrm{f}$ in the turbulent flow regime $3000 \leq \mathrm{Re} \leq 200000$ (which includes the range of $\mathrm{Re}$ realized in this study), the Blasius equation [5]:

$$
f=0.079 \operatorname{Re}^{-0.25}
$$

is found useful in determining $\mathrm{f}$ for use in equation 2 . 


\subsection{Calculation of Loss through Duct Fittings}

For a composite duct run, the total loss due to fittings is given as [4]:

$$
h_{\text {fittings }}=0.08256 \sum_{j=1}^{j=m} k_{j} q_{j}^{2} d_{j}^{-4}
$$

where $k$ is the head loss coefficient of the particular type of fitting [6]; $\mathrm{j}$ denotes the $j^{\text {th }}$ fitting and $m$ is the number of fittings in the composite run; $q$ and $d$ are as defined earlier.

Furthermore, the head loss through duct enlargements is given as [4]:

$$
h_{e}=\frac{8 k_{e}}{\pi^{2} g}\left(\frac{1}{d_{1}{ }^{2}}-\frac{1}{d_{2}{ }^{2}}\right)^{2} q^{2}
$$

where $k_{\mathrm{e}}$ is the head loss coefficient through the enlargement. $d_{1}$ and $d_{2}$ are, respectively, the upstream and downstream diameters at the enlargement. Values of $k_{\mathrm{e}}$ for various values of $d_{2} / d_{1}$ and for various conical angles of enlargement are given in the literature [6]. Figure 3 illustrates the fittings.

Tables 1 and 2 give values of $k$ for radius elbows and tees, and in order to achieve reduced head losses through fittings and to achieve uniformity of flow parameters (for the sake of proper comparison of results), $90^{\circ}$ elbows and radius tees are utilized, while the enlargements are made of $30^{\circ}$ conical angle. Thus, for the different values of $d_{2} / d_{1}$ the respective values of $\mathrm{k}_{\mathrm{e}}$ are obtained from the graphs of Figure 4 [6].

Table 1. Head loss coefficients $\mathrm{k}$ for radius elbows [6].

\begin{tabular}{|c|c|}
\hline$R / D$ & $k$ \\
\hline 0 & 0.8 \\
0.25 & 0.4 \\
0.5 & 0.25 \\
1.0 & 0.16 \\
\hline
\end{tabular}

Table 2. Head loss coefficients $\mathrm{k}$ for radius tees [6].

\begin{tabular}{|c|c|c|}
\hline$R / D$ & $\alpha=90^{\circ}$ & $\alpha=45^{\circ}$ \\
\hline 0 & 1.0 & 0.6 \\
0.25 & 0.5 & 0.35 \\
0.5 & 0.3 & 0.2 \\
1.0 & 0.2 & 0.15 \\
\hline
\end{tabular}

a)
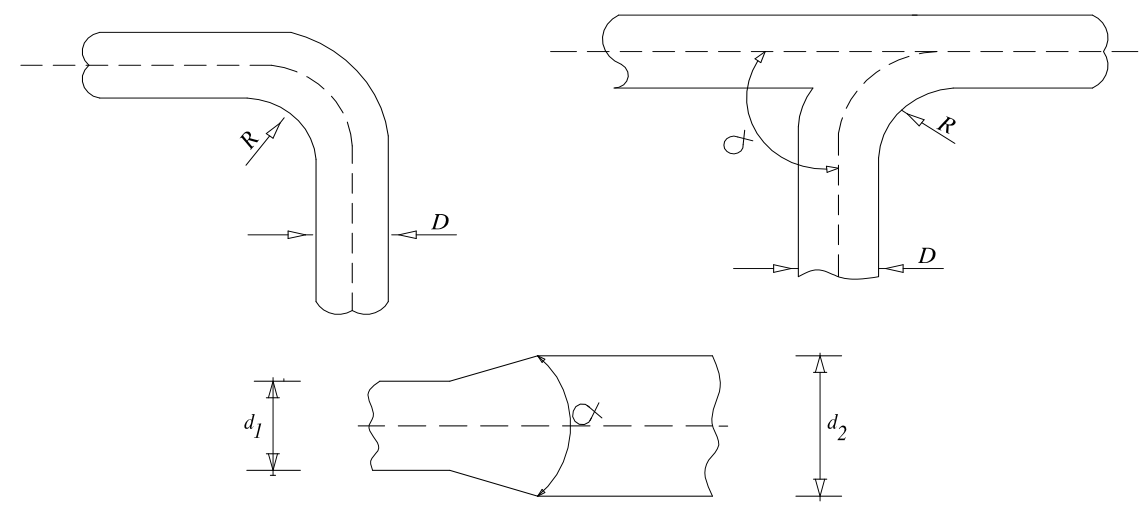

b)

c)

Fig. 3. Illustration of duct fittings: a - Radius elbow; b - Radius tee; c - Duct enlargement. 


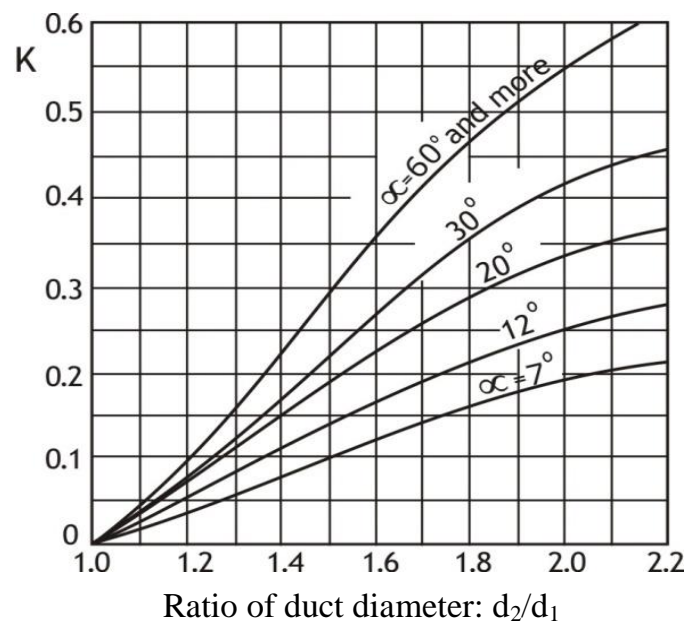

Fig. 4. Head loss coefficients k across duct enlargement for various enlargement angles.

\section{CALCULATION OF HEAD LOSS COMPONENTS FOR THE FIRST INDEX DUCT RUN 0, 1, 2, $\ldots, 12$}

The loss components in the index run $0,1,2,---, 12$ are calculated as an illustration of the general procedure for the other runs listed in section 2 above.

\subsection{Air Quantities}

Using the recommended ventilation requirements stated earlier and the estimated numbers of users or the floor area for each room (as required), the ventilation air quantities are obtained. Hence, with the chosen number of air inlet terminals, the air quantity per inlet is obtained. Table 3 summarizes the calculation of the air quantities. Hence, the cumulative quantity for each air duct section is obtained and shown in Figure 2.

\subsection{A Typical Head Loss Calculation}

The head loss calculations are illustrated as follows using duct section 10-11. Air quantity in duct section, $\mathrm{q}=3.88 \mathrm{~m}^{3} / \mathrm{min}=0.065 \mathrm{~m}^{3} / \mathrm{s}$, duct diameter (from Equation 1), $\mathrm{d}=0.115 \mathrm{~m}$.

Thus, a standard $100 \mathrm{~mm}$ size is taken.

$$
\begin{aligned}
& \operatorname{Re}=8.5154 \times 10^{4} \times \frac{0.065}{0.1}=55347.5 \\
& f=0.079 \times 55347.5^{-0.25}=0.0052
\end{aligned}
$$

Length $l$ of duct section $=3.9 \mathrm{~m}$.

$$
h_{\text {friction }}=\frac{0.3304 \times 0.0052 \times 3.9 \times 0.065^{2}}{0.1^{5}}=2.83 \mathrm{~m}
$$

Now, there is one $100 \mathrm{~mm} \times 150 \mathrm{~mm}$ enlargement, one elbow and one radius tee in this duct section. From Tables 1 and 2 the respective $k$ values for the elbow and radius tee are 0.16 and 0.2 . For the enlargement, $\frac{d_{2}}{d_{1}}=\frac{150}{100}=1.5$ and $k_{e}$ for the $30^{\circ}$ conical angle from Figure 4 is 0.23 .

Then loss through the $100 \mathrm{~mm}$ elbow: 


$$
\mathrm{h}_{\text {fitings }}=0.16 \times 0.08256 \times 0.065^{2} \times 0.1^{-4}=0.558 \mathrm{~m}
$$

Loss through the $150 \mathrm{~mm}$ tee:

$$
\mathrm{h}_{\text {fitings }}=0.2 \times 0.08256 \times 0.065^{2} \times 0.15^{-4}=0.138 \mathrm{~m}
$$

Loss through the $100 \mathrm{~mm} \times 150 \mathrm{~mm}$ enlargement:

$$
h_{e}=\frac{8 \times 0.23}{\pi^{2} \times 9.81}\left(\frac{1}{0.1^{2}}-\frac{1}{0.15^{2}}\right)^{2} 0.065^{2}=0.235 m
$$

Following a similar procedure as for duct section 10-11, all other duct runs are analysed. Table 4 gives the complete computation of frictional and fitting loss components in the index duct run $0,1,2,---, 12$. Thus, the total frictional loss in the index run is $6.275 \mathrm{~m}$ while the total loss through duct fittings is $3.896 \mathrm{~m}$.

Table 3. Calculation of ventilation air quantities.

\begin{tabular}{|l|l|c|c|c|c|c|}
\hline $\begin{array}{c}\text { Room } \\
\text { Type }\end{array}$ & Room Designation & $\begin{array}{c}\text { Estimated No. } \\
\text { of Persons } \\
\text { (where } \\
\text { Required) }\end{array}$ & $\begin{array}{c}\text { Floor Area, } \\
\text { (where } \\
\text { Required) } \mathrm{m}^{2}\end{array}$ & $\begin{array}{c}\text { Ventilation Air } \\
\text { Quantity, } \mathrm{m}^{3} / \mathrm{min}\end{array}$ & $\begin{array}{c}\text { No. of } \\
\text { Ventilation } \\
\text { Duct Inlets } \\
\text { in Room }\end{array}$ & $\begin{array}{c}\text { Air } \\
\text { Quantity } \\
\text { per Inlet, } \\
\mathrm{m}^{3} / \mathrm{min}\end{array}$ \\
\hline Cafeteria & Staff Canteen & 40 & - & $0.34 \times 40=13.60$ & 7 & 1.94 \\
\hline Toilet & Cleaner's Store & - & 4.00 & $0.60 \times 4.00=2.40$ & 1 & 2.40 \\
& Kitchen Staff Toilets & - & 5.00 & $0.60 \times 5.00=3.00$ & 2 & 1.50 \\
& Male Ablution and \\
& Changing Rooms & - & 29.50 & $0.60 \times 29.50=17.70$ & 7 & 2.53 \\
& Female Ablution and \\
& Changing Rooms & - & 29.50 & $0.60 \times 29.50=17.70$ & 7 & 2.53 \\
\hline Kitchen & Kitchen/Wash-up & - & 14.44 & $1.2 \times 14.44=17.33$ & 2 & 8.67 \\
\hline
\end{tabular}

Table 4. Head loss computations for index run 'a' $(0,1,2,-\cdots 12)$.

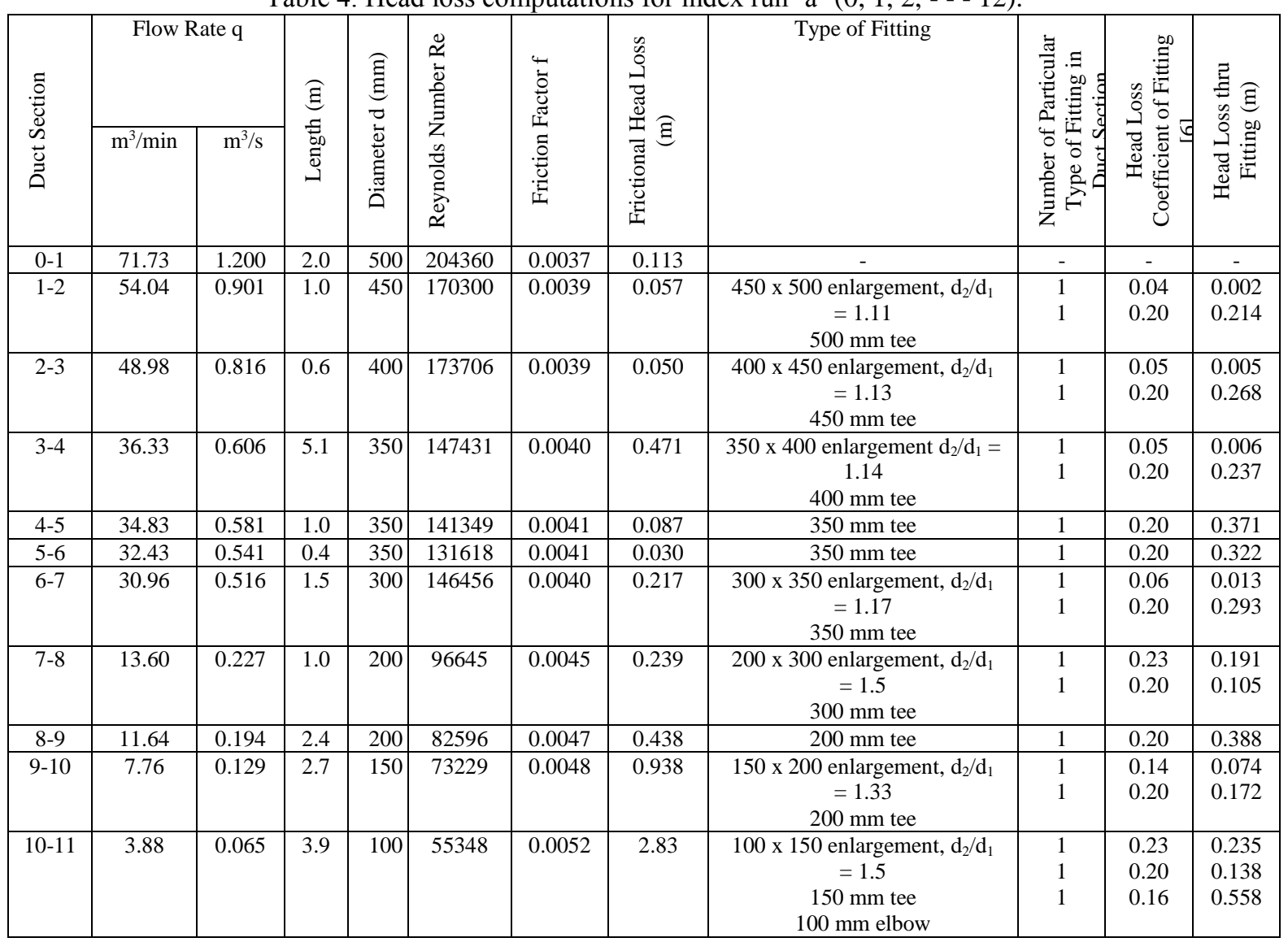




\begin{tabular}{|l|l|l|l|l|l|l|l|l|l|l|l|}
\hline $11-12$ & 1.94 & 0.032 & 3.9 & 100 & 27248 & 0.0061 & 0.305 & $100 \mathrm{~mm}$ tee & 1 & 0.20 & 0.169 \\
& & & & & & & & $100 \mathrm{~mm}$ elbow & 1 & 0.16 & 0.135 \\
\hline & & & 25.5 & & & & 6.275 & & & & 3.896 \\
\hline
\end{tabular}

Table 5. Head loss computations for index run 'b' $(0,1,2,---, 10,13,14)$.

\begin{tabular}{|c|c|c|c|c|c|c|c|c|c|c|c|}
\hline \multirow[b]{2}{*}{ 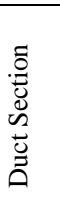 } & \multicolumn{2}{|c|}{ Flow Rate q } & \multirow[b]{2}{*}{ 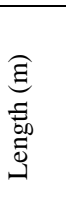 } & \multirow[b]{2}{*}{ 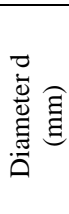 } & \multirow[b]{2}{*}{ 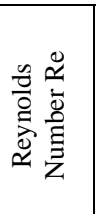 } & \multirow[b]{2}{*}{ 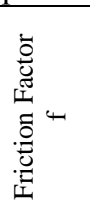 } & \multirow[b]{2}{*}{ 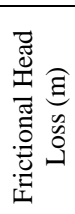 } & \multirow[t]{2}{*}{ Type of Fitting } & \multirow[b]{2}{*}{ 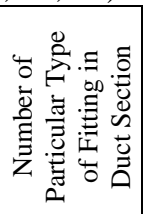 } & \multirow[b]{2}{*}{ 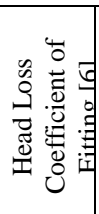 } & \multirow[b]{2}{*}{ 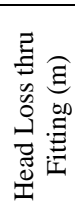 } \\
\hline & $\mathrm{m}^{3} / \mathrm{min}$ & $\mathrm{m}^{3} / \mathrm{s}$ & & & & & & & & & \\
\hline $0-1$ & 71.73 & 1.200 & 2.0 & 500 & 204360 & 0.0037 & 0.113 & - & - & - & - \\
\hline $1-2$ & 54.04 & 0.901 & 1.0 & 450 & 170300 & 0.0039 & 0.057 & $\begin{array}{c}450 \times 500 \text { enlargement, } \mathrm{d}_{2} / \mathrm{d}_{1}= \\
1.11 \\
500 \mathrm{~mm} \text { tee }\end{array}$ & $\begin{array}{l}1 \\
1\end{array}$ & $\begin{array}{l}0.04 \\
0.20\end{array}$ & $\begin{array}{l}0.002 \\
0.214\end{array}$ \\
\hline $2-3$ & 48.98 & 0.816 & 0.6 & 400 & 173706 & 0.0039 & 0.050 & $\begin{array}{c}400 \times 450 \text { enlargement, } \mathrm{d}_{2} / \mathrm{d}_{1}= \\
1.13 \\
450 \mathrm{~mm} \text { tee }\end{array}$ & $\begin{array}{l}1 \\
1\end{array}$ & $\begin{array}{l}0.05 \\
0.20\end{array}$ & $\begin{array}{l}0.005 \\
0.268\end{array}$ \\
\hline $3-4$ & 36.33 & 0.606 & 5.1 & 350 & 147431 & 0.0040 & 0.471 & $\begin{array}{c}350 \times 400 \text { enlargement, } \mathrm{d}_{2} / \mathrm{d}_{1}= \\
1.14 \\
400 \mathrm{~mm} \text { tee }\end{array}$ & $\begin{array}{l}1 \\
1\end{array}$ & $\begin{array}{l}0.05 \\
0.20\end{array}$ & $\begin{array}{l}0.006 \\
0.237\end{array}$ \\
\hline $4-5$ & 34.83 & 0.581 & 1.0 & 350 & 141349 & 0.0041 & 0.087 & $350 \mathrm{~mm}$ tee & 1 & 0.20 & 0.371 \\
\hline $5-6$ & 32.43 & 0.541 & 0.4 & 350 & 131618 & 0.0041 & 0.030 & $350 \mathrm{~mm}$ tee & 1 & 0.20 & 0.322 \\
\hline $6-7$ & 30.96 & 0.516 & 1.5 & 300 & 146456 & 0.0040 & 0.217 & $\begin{array}{c}300 \times 350 \text { enlargement, } \mathrm{d}_{2} / \mathrm{d}_{1}= \\
1.17 \\
350 \mathrm{~mm} \text { tee }\end{array}$ & $\begin{array}{l}1 \\
1\end{array}$ & $\begin{array}{l}0.06 \\
0.20\end{array}$ & $\begin{array}{l}0.013 \\
0.293\end{array}$ \\
\hline $7-8$ & 13.60 & 0.227 & 1.0 & 200 & 96645 & 0.0045 & 0.239 & $\begin{array}{c}200 \times 300 \text { enlargement, } \mathrm{d}_{2} / \mathrm{d}_{1}= \\
1.5 \\
500 \mathrm{~mm} \text { tee }\end{array}$ & $\begin{array}{l}1 \\
1\end{array}$ & $\begin{array}{l}0.23 \\
0.20\end{array}$ & $\begin{array}{l}0.191 \\
0.105\end{array}$ \\
\hline $8-9$ & 11.64 & 0.194 & 2.4 & 200 & 82596 & 0.0047 & 0.438 & $200 \mathrm{~mm}$ tee & 1 & 0.20 & 0.388 \\
\hline $9-10$ & 7.76 & 0.127 & 2.7 & 150 & 73229 & 0.0048 & 0.938 & $\begin{array}{c}150 \times 200 \text { enlargement, } \mathrm{d}_{2} / \mathrm{d}_{1}= \\
1.33 \\
200 \mathrm{~mm} \text { tee }\end{array}$ & $\begin{array}{l}1 \\
1\end{array}$ & $\begin{array}{l}0.14 \\
0.20\end{array}$ & $\begin{array}{l}0.074 \\
0.172\end{array}$ \\
\hline $\begin{array}{l}10- \\
13\end{array}$ & 3.88 & 0.065 & 1.2 & 100 & 55348 & 0.0052 & 0.871 & $\begin{array}{c}100 \times 150 \text { enlargement, } \mathrm{d}_{2} / \mathrm{d}_{1}= \\
1.5 \\
100 \mathrm{~mm} \text { elbow }\end{array}$ & $\begin{array}{l}1 \\
1\end{array}$ & $\begin{array}{l}0.23 \\
0.16\end{array}$ & $\begin{array}{l}0.235 \\
0.558\end{array}$ \\
\hline $\begin{array}{l}13- \\
14\end{array}$ & 1.94 & 0.032 & 3.6 & 100 & 27248 & 0.0061 & 0.743 & $\begin{array}{c}500 \mathrm{~mm} \text { tee } \\
100 \mathrm{~mm} \text { elbow }\end{array}$ & $\begin{array}{l}1 \\
1\end{array}$ & $\begin{array}{l}0.20 \\
0.16\end{array}$ & $\begin{array}{l}0.034 \\
0.135\end{array}$ \\
\hline & & & 22.5 & & & & 4.254 & & & & 3.623 \\
\hline
\end{tabular}

Table 6. Head loss computations for index run 'c' $(0,1,2,---, 9,15,16)$.

\begin{tabular}{|c|c|c|c|c|c|c|c|c|c|c|c|}
\hline \multirow[b]{2}{*}{ 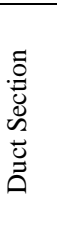 } & \multicolumn{2}{|c|}{ Flow Rate q } & \multirow[b]{2}{*}{ 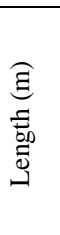 } & \multirow[b]{2}{*}{ 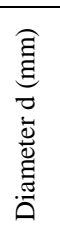 } & \multirow[b]{2}{*}{ 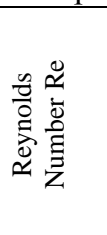 } & \multirow[b]{2}{*}{ 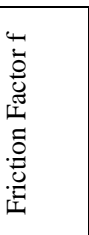 } & \multirow[b]{2}{*}{ 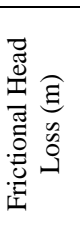 } & \multirow[t]{2}{*}{ Type of Fitting } & \multirow[b]{2}{*}{ 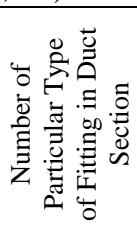 } & \multirow[b]{2}{*}{ 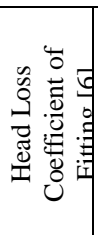 } & \multirow[b]{2}{*}{ 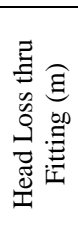 } \\
\hline & $\mathrm{m}^{3} / \mathrm{min}$ & $\mathrm{m}^{3} / \mathrm{s}$ & & & & & & & & & \\
\hline $0-1$ & 71.73 & 1.200 & 2.0 & 500 & 204360 & 0.0037 & 0.113 & - & - & - & - \\
\hline $1-2$ & 54.04 & 0.901 & 1.0 & 450 & 170300 & 0.0039 & 0.057 & $\begin{array}{c}450 \times 500 \text { enlargement, } \\
\mathrm{d}_{2} / \mathrm{d}_{1}=1.11 \\
500 \mathrm{~mm} \text { tee }\end{array}$ & $\begin{array}{l}1 \\
1\end{array}$ & $\begin{array}{l}0.04 \\
0.20\end{array}$ & $\begin{array}{l}0.002 \\
0.214\end{array}$ \\
\hline $2-3$ & 48.98 & 0.816 & 0.6 & 400 & 173706 & 0.0039 & 0.050 & $\begin{array}{c}400 \times 450 \text { enlargement, } \\
\mathrm{d}_{2} / \mathrm{d}_{1}=1.13 \\
450 \mathrm{~mm} \text { tee }\end{array}$ & $\begin{array}{l}1 \\
1\end{array}$ & $\begin{array}{l}0.05 \\
0.20\end{array}$ & $\begin{array}{l}0.005 \\
0.268\end{array}$ \\
\hline $3-4$ & 36.33 & 0.606 & 5.1 & 350 & 147431 & 0.0040 & 0.471 & $\begin{array}{c}350 \times 400 \text { enlargement, } \\
\mathrm{d}_{2} / \mathrm{d}_{1}=1.14 \\
400 \mathrm{~mm} \text { tee }\end{array}$ & $\begin{array}{l}1 \\
1\end{array}$ & $\begin{array}{l}0.05 \\
0.20\end{array}$ & $\begin{array}{l}0.006 \\
0.237\end{array}$ \\
\hline $4-5$ & 34.83 & 0.581 & 1.0 & 350 & 141349 & 0.0041 & 0.087 & $350 \mathrm{~mm}$ tee & 1 & 0.20 & 0.371 \\
\hline $5-6$ & 32.43 & 0.541 & 0.4 & 350 & 131618 & 0.0041 & 0.030 & $350 \mathrm{~mm}$ tee & 1 & 0.20 & 0.322 \\
\hline $6-7$ & 30.96 & 0.516 & 1.5 & 300 & 146456 & 0.0040 & 0.217 & $\begin{array}{c}300 \times 350 \text { enlargement, } \\
\mathrm{d}_{2} / \mathrm{d}_{1}=1.17 \\
350 \mathrm{~mm} \text { tee }\end{array}$ & $\begin{array}{l}1 \\
1\end{array}$ & $\begin{array}{l}0.06 \\
0.20\end{array}$ & $\begin{array}{l}0.013 \\
0.293\end{array}$ \\
\hline $7-8$ & 13.60 & 0.227 & 1.0 & 200 & 96645 & 0.0045 & 0.239 & $\begin{array}{c}200 \times 300 \text { enlargement, } \\
\mathrm{d}_{2} / \mathrm{d}_{1}=1.5 \\
300 \mathrm{~mm} \text { tee } \\
\end{array}$ & $\begin{array}{l}1 \\
1\end{array}$ & $\begin{array}{l}0.23 \\
0.20\end{array}$ & $\begin{array}{l}0.191 \\
0.105\end{array}$ \\
\hline $8-9$ & 11.64 & 0.194 & 2.4 & 200 & 82596 & 0.0047 & 0.438 & $200 \mathrm{~mm}$ tee & 1 & 0.20 & 0.388 \\
\hline
\end{tabular}




\begin{tabular}{|c|c|c|c|c|c|c|c|c|c|c|c|}
\hline $9-15$ & 3.88 & 0.065 & 1.2 & 100 & 55348 & 0.0052 & 0.871 & $\begin{array}{c}100 \times 200 \text { enlargement, } \\
\mathrm{d}_{2} / \mathrm{d}_{1}=2 \\
200 \mathrm{~mm} \text { tee }\end{array}$ & $\begin{array}{l}1 \\
1\end{array}$ & $\begin{array}{l}0.42 \\
0.20\end{array}$ & $\begin{array}{l}0.781 \\
0.044\end{array}$ \\
\hline $\begin{array}{c}15- \\
16\end{array}$ & 1.94 & 0.032 & 3.6 & 100 & 27248 & 0.0061 & 0.743 & $\begin{array}{c}100 \mathrm{~mm} \text { tee } \\
100 \mathrm{~mm} \text { elbow }\end{array}$ & $\begin{array}{l}1 \\
1\end{array}$ & $\begin{array}{l}0.20 \\
0.16\end{array}$ & $\begin{array}{l}0.169 \\
0.135\end{array}$ \\
\hline & & & 19.8 & & & & 3.316 & & & & 3.544 \\
\hline
\end{tabular}

Table 7. Head loss computations for index run'd' $(0,1,2,---, 7,17,18)$

\begin{tabular}{|c|c|c|c|c|c|c|c|c|c|c|c|}
\hline \multirow[b]{2}{*}{ 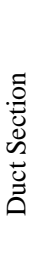 } & \multicolumn{2}{|c|}{ Flow Rate q } & \multirow[b]{2}{*}{ 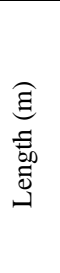 } & \multirow[b]{2}{*}{ 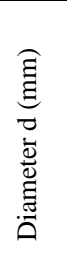 } & \multirow[b]{2}{*}{ 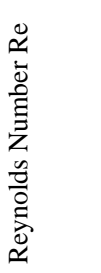 } & \multirow[b]{2}{*}{ 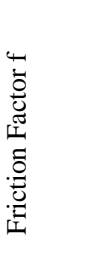 } & \multirow[b]{2}{*}{ 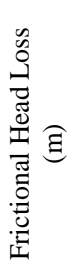 } & \multirow[t]{2}{*}{ Type of Fitting } & \multirow[b]{2}{*}{ 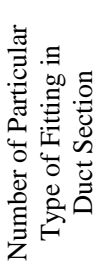 } & \multirow[b]{2}{*}{ 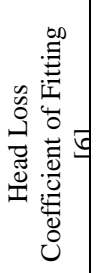 } & \multirow[b]{2}{*}{ 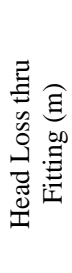 } \\
\hline & $\mathrm{m}^{3} / \mathrm{min}$ & $\mathrm{m}^{3} / \mathrm{s}$ & & & & & & & & & \\
\hline $0-1$ & 71.73 & 1.200 & 2.0 & 500 & 204360 & 0.0037 & 0.113 & - & - & - & - \\
\hline $1-2$ & 54.04 & 0.901 & 1.0 & 450 & 170300 & 0.0039 & 0.057 & $\begin{array}{c}450 \times 500 \text { enlargement, } \mathrm{d}_{2} / \mathrm{d}_{1}= \\
1.11 \\
500 \mathrm{~mm} \text { tee }\end{array}$ & $\begin{array}{l}1 \\
1\end{array}$ & $\begin{array}{l}0.04 \\
0.20\end{array}$ & $\begin{array}{l}0.002 \\
0.214\end{array}$ \\
\hline $2-3$ & 48.98 & 0.816 & 0.6 & 400 & 173706 & 0.0039 & 0.050 & $\begin{array}{c}400 \times 450 \text { enlargement, } \mathrm{d}_{2} / \mathrm{d}_{1}= \\
1.13 \\
450 \mathrm{~mm} \text { tee }\end{array}$ & $\begin{array}{l}1 \\
1\end{array}$ & $\begin{array}{l}0.05 \\
0.20\end{array}$ & $\begin{array}{l}0.005 \\
0.268\end{array}$ \\
\hline $3-4$ & 36.33 & 0.606 & 5.1 & 350 & 147431 & 0.0040 & 0.471 & $\begin{array}{c}350 \times 400 \text { enlargement, } \mathrm{d}_{2} / \mathrm{d}_{1}= \\
1.14 \\
400 \mathrm{~mm} \text { tee }\end{array}$ & $\begin{array}{l}1 \\
1\end{array}$ & $\begin{array}{l}0.05 \\
0.20\end{array}$ & $\begin{array}{l}0.006 \\
0.237\end{array}$ \\
\hline $4-5$ & 34.83 & 0.581 & 1.0 & 350 & 141349 & 0.0041 & 0.087 & $350 \mathrm{~mm}$ tee & 1 & 0.20 & 0.371 \\
\hline $5-6$ & 32.43 & 0.541 & 0.4 & 350 & 131618 & 0.0041 & 0.030 & $350 \mathrm{~mm}$ tee & 1 & 0.20 & 0.322 \\
\hline $6-7$ & 30.93 & 0.516 & 1.5 & 300 & 146456 & 0.0040 & 0.217 & $\begin{array}{c}300 \times 350 \text { enlargement, } \mathrm{d}_{2} / \mathrm{d}_{1}= \\
1.17 \\
350 \mathrm{~mm} \text { tee }\end{array}$ & $\begin{array}{l}1 \\
1\end{array}$ & $\begin{array}{l}0.06 \\
0.20\end{array}$ & $\begin{array}{l}0.013 \\
0.293\end{array}$ \\
\hline $\begin{array}{l}7- \\
17\end{array}$ & 17.33 & 0.289 & 3.8 & 250 & 98433 & 0.0045 & 0.483 & $\begin{array}{c}250 \times 300 \text { enlargement, } \mathrm{d}_{2} / \mathrm{d}_{1}= \\
1.2 \\
300 \mathrm{~mm} \text { tee }\end{array}$ & $\begin{array}{l}1 \\
1\end{array}$ & $\begin{array}{l}0.08 \\
0.20\end{array}$ & $\begin{array}{l}0.113 \\
0.170\end{array}$ \\
\hline $\begin{array}{l}17- \\
18\end{array}$ & 8.67 & 0.145 & 1.7 & 150 & 82312 & 0.0047 & 0.731 & $\begin{array}{c}150 \times 250 \text { enlargement, } \mathrm{d}_{2} / \mathrm{d}_{1}= \\
1.67 \\
250 \mathrm{~mm} \text { tee } \\
150 \mathrm{~mm} \text { elbow } \\
\end{array}$ & $\begin{array}{l}1 \\
1 \\
1\end{array}$ & $\begin{array}{l}0.30 \\
0.20 \\
0.16\end{array}$ & $\begin{array}{l}0.210 \\
0.089 \\
0.549\end{array}$ \\
\hline & & & 17.1 & & & & 2.239 & & & & 2.762 \\
\hline
\end{tabular}

Table 8. Head loss computations for index run 'e' $(0,1,2,-\cdots, 5,19)$.

\begin{tabular}{|c|c|c|c|c|c|c|c|c|c|c|c|}
\hline \multirow[b]{2}{*}{ 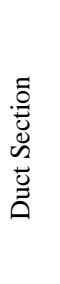 } & \multicolumn{2}{|c|}{ Flow Rate q } & \multirow[b]{2}{*}{ 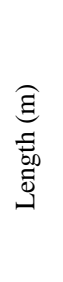 } & \multirow[b]{2}{*}{ 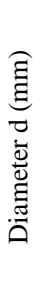 } & \multirow[b]{2}{*}{ 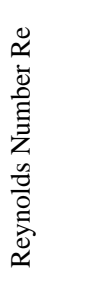 } & \multirow[b]{2}{*}{ 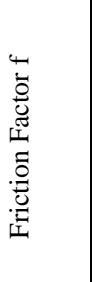 } & \multirow[b]{2}{*}{ 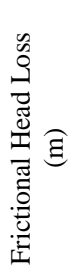 } & \multirow[t]{2}{*}{ Type of Fitting } & \multirow[b]{2}{*}{ 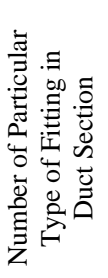 } & \multirow[b]{2}{*}{ 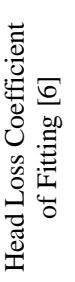 } & \multirow[b]{2}{*}{ 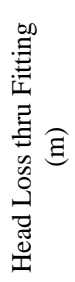 } \\
\hline & $\mathrm{m}^{3} / \mathrm{min}$ & $\mathrm{m}^{3} / \mathrm{s}$ & & & & & & & & & \\
\hline $0-1$ & 71.73 & 1.200 & 2.0 & 500 & 204360 & 0.0037 & 0.113 & - & - & - & - \\
\hline $1-2$ & 54.04 & 0.901 & 1.0 & 450 & 170300 & 0.0039 & 0.057 & $\begin{array}{c}450 \times 500 \text { enlargement, } \mathrm{d}_{2} / \mathrm{d}_{1}= \\
1.11 \\
500 \mathrm{~mm} \text { tee }\end{array}$ & $\begin{array}{l}1 \\
1\end{array}$ & $\begin{array}{l}0.04 \\
0.20\end{array}$ & $\begin{array}{l}0.002 \\
0.214\end{array}$ \\
\hline $2-3$ & 48.98 & 0.816 & 0.6 & 400 & 173706 & 0.0039 & 0.050 & $\begin{array}{c}400 \times 450 \text { enlargement, } \mathrm{d}_{2} / \mathrm{d}_{1}= \\
1.13 \\
450 \mathrm{~mm} \text { tee }\end{array}$ & $\begin{array}{l}1 \\
1\end{array}$ & $\begin{array}{l}0.05 \\
0.20\end{array}$ & $\begin{array}{l}0.005 \\
0.268\end{array}$ \\
\hline $3-4$ & 36.33 & 0.606 & 5.1 & 350 & 147431 & 0.0040 & 0.471 & $\begin{array}{c}350 \times 400 \text { enlargement, } \mathrm{d}_{2} / \mathrm{d}_{1}= \\
1.14 \\
400 \mathrm{~mm} \text { tee }\end{array}$ & $\begin{array}{l}1 \\
1\end{array}$ & $\begin{array}{l}0.05 \\
0.20\end{array}$ & $\begin{array}{l}0.006 \\
0.237\end{array}$ \\
\hline $4-5$ & 34.83 & 0.581 & 1.0 & 350 & 141349 & 0.0041 & 0.087 & $350 \mathrm{~mm}$ tee & 1 & 0.20 & 0.371 \\
\hline $5-19$ & 2.40 & 0.040 & 3.8 & 100 & 34060 & 0.0058 & 1.17 & $\begin{array}{c}100 \times 200 \text { enlargement, } \mathrm{d}_{2} / \mathrm{d}_{1}= \\
2.0 \\
200 \times 350 \text { enlargement, } \mathrm{d}_{2} / \mathrm{d}_{1}= \\
1.75 \\
350 \mathrm{~mm} \text { tee } \\
100 \mathrm{~mm} \text { elbow } \\
\end{array}$ & $\begin{array}{l}1 \\
1 \\
1 \\
1\end{array}$ & $\begin{array}{l}0.42 \\
0.34 \\
0.20 \\
0.16\end{array}$ & $\begin{array}{l}0.390 \\
0.016 \\
0.002 \\
0.211\end{array}$ \\
\hline & & & 13.5 & & & & 1.948 & & & & 1.722 \\
\hline
\end{tabular}


Table 9. Head loss computations for index run ' $\mathrm{f}$ ' $(0,1,2,3,20,---, 24)$.

\begin{tabular}{|c|c|c|c|c|c|c|c|c|c|c|c|}
\hline \multirow[b]{2}{*}{ 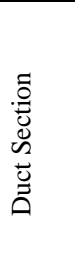 } & \multicolumn{2}{|c|}{ Flow Rate q } & \multirow[b]{2}{*}{ 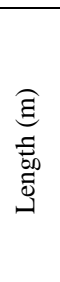 } & \multirow[b]{2}{*}{ 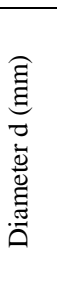 } & \multirow[b]{2}{*}{ 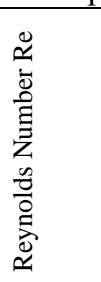 } & \multirow[b]{2}{*}{ 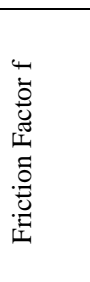 } & \multirow[b]{2}{*}{ 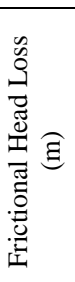 } & \multirow[t]{2}{*}{ Type of Fitting } & \multirow[b]{2}{*}{ 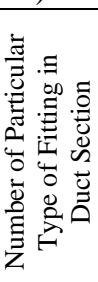 } & \multirow[b]{2}{*}{ 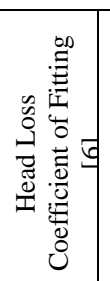 } & \multirow[b]{2}{*}{ 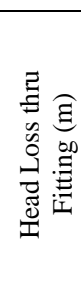 } \\
\hline & $\begin{array}{c}\mathrm{m}^{3} / \mathrm{mi} \\
\mathrm{n}\end{array}$ & $\mathrm{m}^{3} / \mathrm{s}$ & & & & & & & & & \\
\hline $0-1$ & 71.73 & 1.200 & 2.0 & 500 & 204360 & 0.0037 & 0.113 & - & - & - & - \\
\hline $1-2$ & 54.04 & 0.901 & 1.0 & 450 & 170300 & 0.0039 & 0.057 & $\begin{array}{c}450 \times 500 \text { enlargement, } \mathrm{d}_{2} / \mathrm{d}_{1}= \\
1.11 \\
500 \mathrm{~mm} \text { tee }\end{array}$ & $\begin{array}{l}1 \\
1\end{array}$ & $\begin{array}{l}0.04 \\
0.20\end{array}$ & $\begin{array}{l}0.002 \\
0.214\end{array}$ \\
\hline $2-3$ & 48.98 & 0.816 & 0.6 & 400 & 173706 & 0.0039 & 0.050 & $\begin{array}{c}400 \times 450 \text { enlargement, } \mathrm{d}_{2} / \mathrm{d}_{1}= \\
1.13 \\
450 \mathrm{~mm} \text { tee }\end{array}$ & $\begin{array}{l}1 \\
1\end{array}$ & $\begin{array}{l}0.05 \\
0.20\end{array}$ & $\begin{array}{l}0.005 \\
0.268\end{array}$ \\
\hline $3-20$ & 12.65 & 0.211 & 0.7 & 200 & 89833 & 0.0046 & 0.148 & $\begin{array}{c}200 \times 400 \text { enlargement, } \mathrm{d}_{2} / \mathrm{d}_{1}= \\
2 \\
400 \mathrm{~mm} \text { tee }\end{array}$ & $\begin{array}{l}1 \\
1\end{array}$ & $\begin{array}{l}0.42 \\
0.20\end{array}$ & $\begin{array}{l}0.549 \\
0.029\end{array}$ \\
\hline $20-21$ & 10.12 & 0.169 & 1.2 & 200 & 71952 & 0.0048 & 0.170 & $200 \mathrm{~mm}$ tee & 1 & 0.20 & 0.295 \\
\hline $21-22$ & 7.59 & 0.127 & 1.3 & 150 & 72094 & 0.0048 & 0.438 & $\begin{array}{c}150 \times 200 \text { enlargement, } \mathrm{d}_{2} / \mathrm{d}_{1}= \\
1.33 \\
200 \mathrm{~mm} \text { tee }\end{array}$ & $\begin{array}{l}1 \\
1\end{array}$ & $\begin{array}{l}0.14 \\
0.20\end{array}$ & $\begin{array}{l}0.070 \\
0.166\end{array}$ \\
\hline $22-23$ & 5.06 & 0.084 & 1.4 & 150 & 47684 & 0.0053 & 0.228 & $150 \mathrm{~mm}$ tee & 1 & 0.20 & 0.230 \\
\hline $23-24$ & 2.53 & 0.042 & $\begin{array}{ll}1.7 \\
\end{array}$ & 100 & 35763 & 0.0057 & 0.565 & $\begin{array}{c}100 \times 150 \text { enlargement, } \mathrm{d}_{2} / \mathrm{d}_{1}= \\
1.5 \\
150 \mathrm{~mm} \text { tee } \\
100 \mathrm{~mm} \text { elbow }\end{array}$ & $\begin{array}{l}1 \\
1 \\
2\end{array}$ & $\begin{array}{l}0.23 \\
0.20 \\
0.16\end{array}$ & $\begin{array}{c}0.009 \\
0.058 \\
0.233 \\
\times 2 \\
=0.46 \\
6\end{array}$ \\
\hline & & & 9.9 & & & & 1.769 & & & & 2.361 \\
\hline
\end{tabular}

Table 10. Head loss computations for index run ' $\mathrm{g}$ ' $(0,1,2,3,20,25)$.

\begin{tabular}{|c|c|c|c|c|c|c|c|c|c|c|c|}
\hline \multirow[b]{2}{*}{ 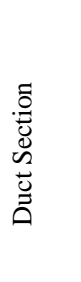 } & \multicolumn{2}{|c|}{ Flow Rate $\mathrm{q}$} & \multirow[b]{2}{*}{ 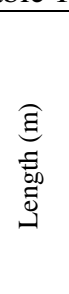 } & \multirow[b]{2}{*}{ 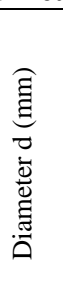 } & \multirow[b]{2}{*}{ 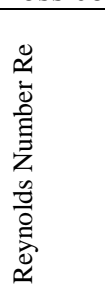 } & \multirow[b]{2}{*}{ 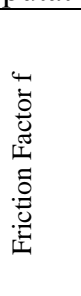 } & \multirow[b]{2}{*}{ 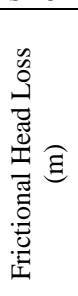 } & \multirow[t]{2}{*}{ Type of Fitting } & \multirow[b]{2}{*}{ 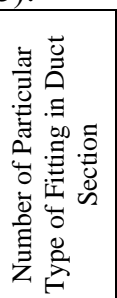 } & \multirow[b]{2}{*}{ 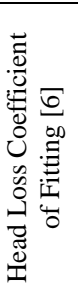 } & \multirow[b]{2}{*}{ 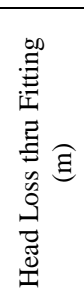 } \\
\hline & $\begin{array}{c}\mathrm{m}^{3} / \mathrm{mi} \\
\mathrm{n}\end{array}$ & $\mathrm{m}^{3} / \mathrm{s}$ & & & & & & & & & \\
\hline $0-1$ & 71.73 & 1.200 & 2.0 & 500 & 204360 & $\begin{array}{c}0.003 \\
7\end{array}$ & 0.113 & - & - & - & - \\
\hline $1-2$ & 54.04 & 0.901 & 1.0 & 450 & 170300 & $\begin{array}{c}0.003 \\
9\end{array}$ & 0.057 & $\begin{array}{c}450 \times 500 \text { enlargement, } \mathrm{d}_{2} / \mathrm{d}_{1}= \\
1.11 \\
500 \mathrm{~mm} \text { tee }\end{array}$ & $\begin{array}{l}1 \\
1\end{array}$ & $\begin{array}{l}0.04 \\
0.20\end{array}$ & $\begin{array}{l}0.002 \\
0.214\end{array}$ \\
\hline $2-3$ & 48.98 & 0.816 & 0.6 & 400 & 173706 & $\begin{array}{c}0.003 \\
9\end{array}$ & 0.050 & $\begin{array}{c}400 \times 450 \text { enlargement, } \mathrm{d}_{2} / \mathrm{d}_{1}= \\
1.13 \\
450 \mathrm{~mm} \text { tee }\end{array}$ & $\begin{array}{l}1 \\
1\end{array}$ & $\begin{array}{l}0.05 \\
0.20\end{array}$ & $\begin{array}{l}0.005 \\
0.268\end{array}$ \\
\hline $3-20$ & 12.65 & 0.211 & 0.7 & 200 & 89833 & $\begin{array}{c}0.004 \\
6\end{array}$ & 0.148 & $\begin{array}{c}200 \times 400 \text { enlargement, } \mathrm{d}_{2} / \mathrm{d}_{1}= \\
2 \\
400 \mathrm{~mm} \text { tee }\end{array}$ & $\begin{array}{l}1 \\
1\end{array}$ & $\begin{array}{l}0.42 \\
0.20\end{array}$ & $\begin{array}{l}0.549 \\
0.029\end{array}$ \\
\hline $20-25$ & 2.53 & 0.042 & 2.3 & 100 & 35763 & $\begin{array}{c}0.005 \\
7\end{array}$ & 0.764 & $\begin{array}{c}100 \times 200 \text { enlargement, } \mathrm{d}_{2} / \mathrm{d}_{1}= \\
2 \\
200 \mathrm{~mm} \text { tee } \\
150 \mathrm{~mm} \text { elbow }\end{array}$ & $\begin{array}{l}1 \\
1 \\
1\end{array}$ & $\begin{array}{l}0.42 \\
0.20 \\
0.16\end{array}$ & $\begin{array}{l}0.390 \\
0.018 \\
0.233\end{array}$ \\
\hline & & & 6.6 & & & & 1.132 & & & & 1.708 \\
\hline
\end{tabular}


Table 11. Head loss computations for index run ' $h$ ' $(0,1,26,---, 31)$.

\begin{tabular}{|c|c|c|c|c|c|c|c|c|c|c|c|}
\hline \multirow[b]{2}{*}{ 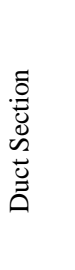 } & \multicolumn{2}{|c|}{ Flow Rate q } & \multirow[b]{2}{*}{ 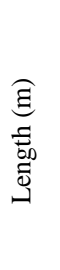 } & \multirow[b]{2}{*}{ 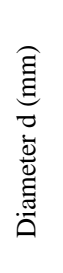 } & \multirow[b]{2}{*}{ 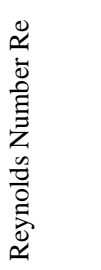 } & \multirow[b]{2}{*}{ 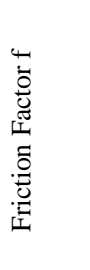 } & \multirow[b]{2}{*}{ 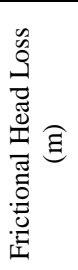 } & \multirow[t]{2}{*}{ Type of Fitting } & \multirow[b]{2}{*}{ 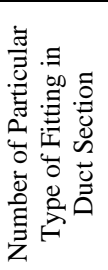 } & \multirow[b]{2}{*}{ 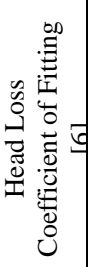 } & \multirow[b]{2}{*}{ 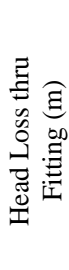 } \\
\hline & $\begin{array}{c}\mathrm{m}^{3} / \mathrm{mi} \\
\mathrm{n}\end{array}$ & $\mathrm{m}^{3} / \mathrm{s}$ & & & & & & & & & \\
\hline $0-1$ & 71.73 & 1.200 & 2.0 & 500 & 204360 & 0.0037 & 0.113 & - & - & - & - \\
\hline $1-2$ & 54.04 & 0.901 & 1.0 & 450 & 170300 & 0.0039 & 0.057 & $\begin{array}{c}450 \times 500 \text { enlargement, } \mathrm{d}_{2} / \mathrm{d}_{1}= \\
1.11 \\
500 \mathrm{~mm} \text { tee }\end{array}$ & $\begin{array}{l}1 \\
1\end{array}$ & $\begin{array}{l}0.04 \\
0.20\end{array}$ & $\begin{array}{l}0.002 \\
0.214\end{array}$ \\
\hline $2-3$ & 48.98 & 0.816 & 0.6 & 400 & 173706 & 0.0039 & 0.050 & $\begin{array}{c}400 \times 450 \text { enlargement, } \mathrm{d}_{2} / \mathrm{d}_{1}= \\
1.13 \\
450 \mathrm{~mm} \text { tee }\end{array}$ & $\begin{array}{l}1 \\
1\end{array}$ & $\begin{array}{l}0.05 \\
0.20\end{array}$ & $\begin{array}{l}0.005 \\
0.268\end{array}$ \\
\hline $1-26$ & 17.70 & 0.295 & 1.0 & 250 & 100477 & 0.0044 & 0.130 & $\begin{array}{c}250 \times 500 \text { enlargement, } \mathrm{d}_{2} / \mathrm{d}_{1}= \\
2 \\
500 \mathrm{~mm} \text { tee }\end{array}$ & $\begin{array}{l}1 \\
1\end{array}$ & $\begin{array}{l}0.42 \\
0.20\end{array}$ & $\begin{array}{l}0.435 \\
0.023\end{array}$ \\
\hline $26-27$ & 12.65 & 0.211 & 1.9 & 200 & 89833 & 0.0046 & 0.402 & $\begin{array}{c}200 \times 250 \text { enlargement, } \mathrm{d}_{2} / \mathrm{d}_{1}= \\
1.25 \\
250 \mathrm{~mm} \text { tee } \\
200 \mathrm{~mm} \text { elbow }\end{array}$ & $\begin{array}{l}1 \\
1 \\
1\end{array}$ & $\begin{array}{l}0.10 \\
0.20 \\
0.16\end{array}$ & $\begin{array}{l}0.030 \\
0.188 \\
0.368\end{array}$ \\
\hline $27-28$ & 10.12 & 0.169 & 0.6 & 200 & 71952 & 0.0048 & 0.085 & $200 \mathrm{~mm}$ tee & 1 & 0.20 & 0.295 \\
\hline $28-29$ & 7.59 & 0.127 & 1.2 & 150 & 72094 & 0.0048 & 0.404 & $\begin{array}{c}150 \times 200 \text { enlargement, } \mathrm{d}_{2} / \mathrm{d}_{1}= \\
1.33 \\
200 \mathrm{~mm} \text { tee }\end{array}$ & $\begin{array}{l}1 \\
1\end{array}$ & $\begin{array}{l}0.14 \\
0.20\end{array}$ & $\begin{array}{l}0.070 \\
0.166\end{array}$ \\
\hline $29-30$ & 5.06 & 0.084 & 1.0 & 150 & 47684 & 0.0053 & 0.163 & $150 \mathrm{~mm}$ tee & 1 & 0.20 & 0.230 \\
\hline $30-31$ & 2.53 & 0.042 & 1.7 & 100 & 35763 & 0.0057 & 0.565 & $\begin{array}{c}100 \times 150 \text { enlargement, } \mathrm{d}_{2} / \mathrm{d}_{1}= \\
1.5 \\
150 \mathrm{~mm} \text { tee } \\
100 \mathrm{~mm} \text { elbow }\end{array}$ & $\begin{array}{l}1 \\
1 \\
1\end{array}$ & $\begin{array}{l}0.23 \\
0.20 \\
0.16\end{array}$ & $\begin{array}{l}0.117 \\
0.058 \\
0.233\end{array}$ \\
\hline & & & 11.0 & & & & 1.969 & & & & 2.702 \\
\hline
\end{tabular}

Table 12. Head loss computations for index run 'i' $(0,1,27,27,32)$.

\begin{tabular}{|c|c|c|c|c|c|c|c|c|c|c|c|}
\hline \multirow[b]{2}{*}{ 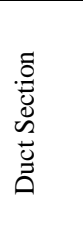 } & \multicolumn{2}{|c|}{ Flow Rate q } & \multirow[b]{2}{*}{ 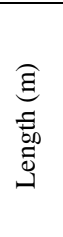 } & \multirow[b]{2}{*}{ 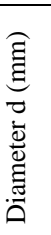 } & \multirow[b]{2}{*}{ 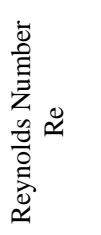 } & \multirow[b]{2}{*}{ 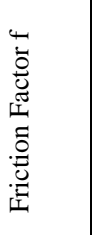 } & \multirow[b]{2}{*}{ 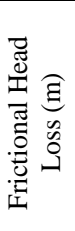 } & \multirow[t]{2}{*}{ Type of Fitting } & \multirow[b]{2}{*}{ 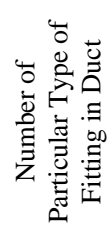 } & \multirow[b]{2}{*}{ 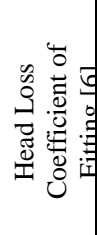 } & \multirow[b]{2}{*}{ 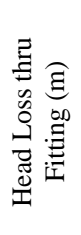 } \\
\hline & $\begin{array}{c}\mathrm{m}^{3} / \mathrm{mi} \\
\mathrm{n}\end{array}$ & $\mathrm{m}^{3} / \mathrm{s}$ & & & & & & & & & \\
\hline $0-1$ & 71.73 & 1.200 & 2.0 & 500 & 204360 & 0.0037 & 0.113 & - & - & - & - \\
\hline $1-26$ & $17-70$ & 0.295 & 1.0 & 250 & 100477 & 0.0044 & 0.130 & $\begin{array}{c}250 \times 500 \text { enlargement, } \mathrm{d}_{2} / \mathrm{d}_{1}= \\
2 \\
500 \mathrm{~mm} \text { tee }\end{array}$ & $\begin{array}{l}1 \\
1\end{array}$ & $\begin{array}{l}0.42 \\
0.20\end{array}$ & $\begin{array}{l}0.390 \\
0.023\end{array}$ \\
\hline $26-27$ & 12.65 & 0.211 & 1.9 & 200 & 89833 & 0.0046 & 0.402 & $\begin{array}{c}200 \times 250 \text { enlargement, } \mathrm{d}_{2} / \mathrm{d}_{1}= \\
1.25 \\
250 \mathrm{~mm} \text { tee } \\
200 \mathrm{~mm} \text { elbow }\end{array}$ & $\begin{array}{l}1 \\
1 \\
1\end{array}$ & $\begin{array}{l}0.10 \\
0.20 \\
0.16\end{array}$ & $\begin{array}{l}0.030 \\
0.188 \\
0.368\end{array}$ \\
\hline $27-32$ & 2.53 & 0.042 & 2.2 & 100 & 35763 & 0.0057 & 0.731 & $\begin{array}{c}100 \times 200 \text { enlargement, } \mathrm{d}_{2} / \mathrm{d}_{1}= \\
2 \\
200 \mathrm{~mm} \text { tee } \\
100 \mathrm{~mm} \text { elbow }\end{array}$ & $\begin{array}{l}1 \\
1 \\
1\end{array}$ & $\begin{array}{l}0.42 \\
0.20 \\
0.16\end{array}$ & $\begin{array}{l}0.390 \\
0.018 \\
0.233\end{array}$ \\
\hline & & & 7.1 & & & & 1.376 & & & & 1.640 \\
\hline
\end{tabular}

Table 13. Summary of head losses.

\begin{tabular}{|c|c|c|c|c|c|}
\hline $\begin{array}{c}\text { Index Run } \\
\text { Designation }\end{array}$ & $\begin{array}{c}\text { Length of } \\
\text { Run }(\mathrm{m})\end{array}$ & $\begin{array}{c}\text { Frictional } \\
\text { Loss }(\mathrm{m})\end{array}$ & $\begin{array}{c}\text { Head Loss } \\
\text { through Fittings } \\
(\mathrm{m})\end{array}$ & $\begin{array}{c}\text { Total Loss } \\
(\mathrm{m})\end{array}$ & $\begin{array}{c}\text { Fraction of } \\
\text { Loss Due to } \\
\text { Fittings }\end{array}$ \\
\hline $\mathrm{a}$ & 25.5 & 6.275 & 3.896 & 10.171 & 0.38 \\
\hline $\mathrm{b}$ & 22.5 & 4.254 & 3.623 & 7.877 & 0.46 \\
\hline $\mathrm{c}$ & 19.8 & 3.316 & 3.544 & 6.860 & 0.52 \\
\hline $\mathrm{d}$ & 17.1 & 2.239 & 2.762 & 5.001 & 0.55 \\
\hline $\mathrm{e}$ & 13.5 & 1.948 & 1.722 & 3.670 & 0.47 \\
\hline $\mathrm{f}$ & 9.9 & 1.769 & 2.361 & 4.130 & 0.57 \\
\hline
\end{tabular}




\begin{tabular}{|c|c|c|c|c|c|}
\hline $\begin{array}{c}\text { Index Run } \\
\text { Designation }\end{array}$ & $\begin{array}{c}\text { Length of } \\
\text { Run }(\mathrm{m})\end{array}$ & $\begin{array}{c}\text { Frictional } \\
\text { Loss }(\mathrm{m})\end{array}$ & $\begin{array}{c}\text { Head Loss } \\
\text { through Fittings } \\
(\mathrm{m})\end{array}$ & $\begin{array}{c}\text { Total Loss } \\
(\mathrm{m})\end{array}$ & $\begin{array}{c}\text { Fraction of } \\
\text { Loss Due to } \\
\text { Fittings }\end{array}$ \\
\hline $\mathrm{g}$ & 6.6 & 1.132 & 1.708 & 2.840 & 0.60 \\
\hline $\mathrm{h}$ & 11.0 & 1.969 & 2.703 & 4.672 & 0.58 \\
\hline $\mathrm{i}$ & 7.1 & 1.376 & 1.640 & 3.016 & 0.54 \\
\hline
\end{tabular}

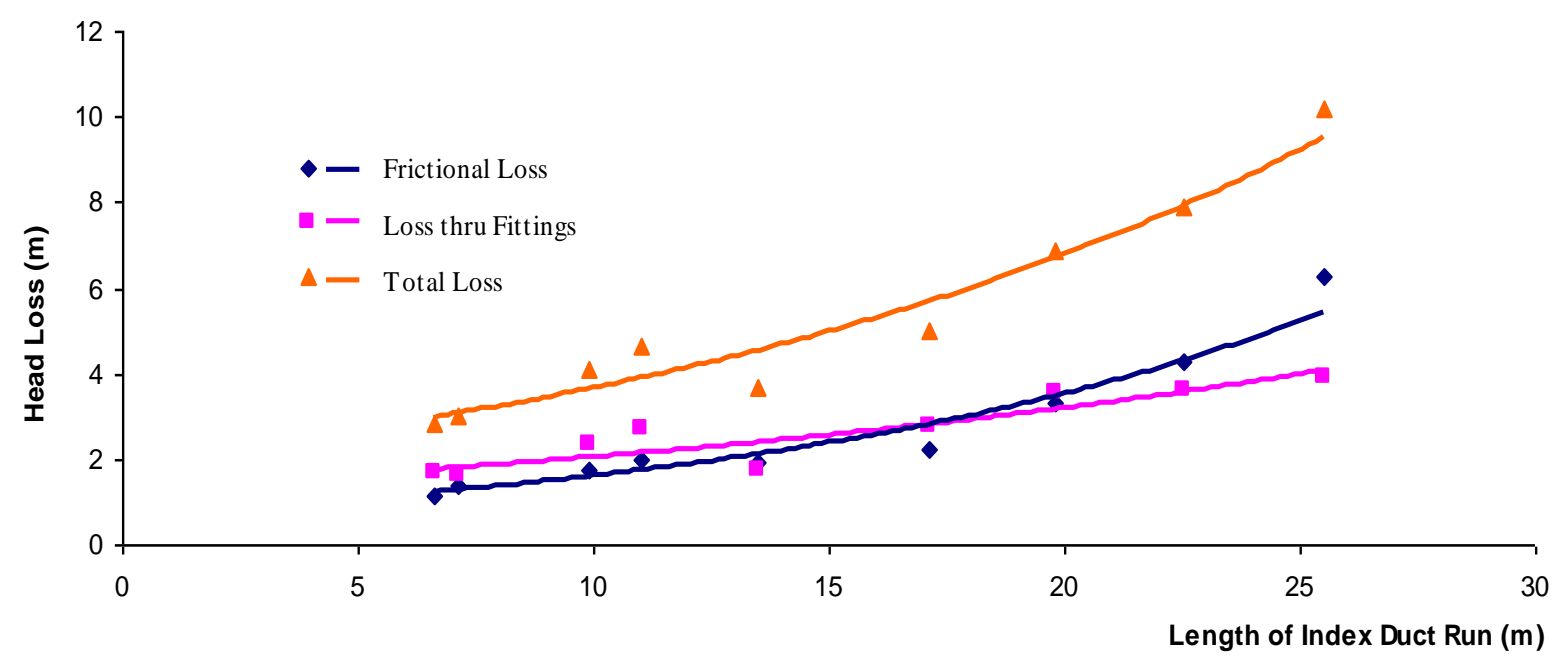

Fig. 5. Variation of Head Losses with Length of Index Run.

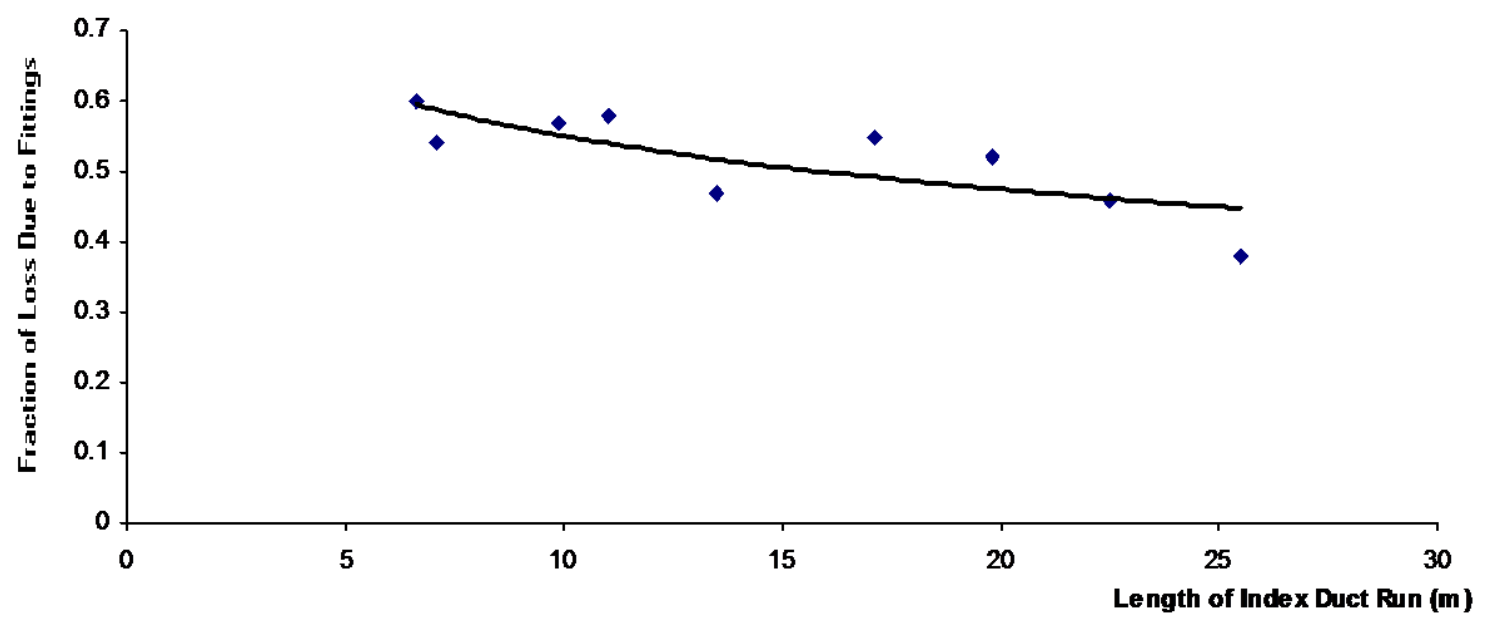

Fig. 6. Variation of Fraction of Loss due to Fittings with Length of Index Run.

\section{RESULTS AND DISCUSSIONS}

Similarly, Tables 5 to 12 give the head loss computations for the other index runs enumerated as ' $b$ ' to ' $i$ ' in section 2 above, while Table 13 gives a summary of the computed losses as well as the fractions of the loss due to duct fittings for the different index runs. The graphs of Figure 5 show general increases of both the frictional loss and the loss due to fittings and, hence, the total system pressure loss with increasing length of index duct run. The increases occur in accordance with equations 2, 6 and 7. Furthermore, the plot of Figure 6 depicts the variation of the fraction of loss due to duct fittings with length of index duct run. This graph shows a general decrease of the fraction of loss due to fittings with increasing length of duct run (with a corresponding increase of the fraction due to friction). This decrease has resulted from the reason that as duct lengths increase, the numbers of duct fittings added into the duct runs are not proportionately increased. 
The fraction of head loss due to duct fittings decreases from 0.60 to 0.45 as the length of duct run increases from $6.6 \mathrm{~m}$ to $25.5 \mathrm{~m}$. Thus, an average value of 0.525 may be utilized to approximate the fraction of loss due to fittings for the range of duct lengths utilized in this study. Hence, having obtained the frictional loss in an index run by the methods illustrated in this paper, the total loss is readily obtained by adding the relevant fraction due to fittings.

In the duct configuration utilized, it is observed that the largest total loss of $10.171 \mathrm{~m}$ occurs in the index run designated as ' $a$ ' in Table 13. This loss in the first index run is, thus, utilized in the extract fan selection [2, 7].

Alternatively, having obtained the frictional loss of $6.275 \mathrm{~m}$ for this duct run, applying the average fraction of 0.525 of the total to account for the loss through fittings gives the total loss through the first index run as follows:

Let the loss through fittings $=x$. Then, $\frac{x}{6.275+x}=0.525$ and $x=6.936 \mathrm{~m}$. Total loss $=6.275+6.936=13.211$

$\mathrm{m}$. This figure, being larger than $10.171 \mathrm{~m}$, gives a margin of safety in extract fan selection.

\section{CONCLUSIONS}

Within the range of lengths of duct run utilized in the study, the frictional and fitting head loss components are comparable in magnitude as can be deduced from Figure 5. It would, therefore, be a misnomer to refer to the fitting loss component as 'minor loss'. This conclusion has also been observed in earlier studies on ventilation and air conditioning ducts $[1,8,9]$.

Representative fractions of head loss through duct fittings, obtained in similar manner to that discussed, for other configurations of extract duct would facilitate loss estimates and extract fan selection. For configurations which are not too different from the one analysed in this paper the results obtained may be applied.

\section{REFERENCES}

[1] Sodiki, J. I., Head loss components in conditioned air branch ducts, International Journal of Academic Research Part A: Applied and Natural Sciences, vol. 6, no. 6, 2014, p. 388 - 394.

[2] Desai, P. S., Modern refrigeration and air conditioning for engineers part II, Khana Publishers, Delhi, 2009.

[3] Institute of Plumbing (IOP), Plumbing services design guide, IOP, Essex, 1977.

[4] Sodiki, J. I., A representative expression for swimming pool circulator pump selection, Nigerian Journal of Engineering Research and Development, vol.1, no. 4, 2002, p. 24 -35.

[5] Kiijavi, J., Darcy friction factor formulae in turbulent pipe flow, Lunova Fluid Mechanics, Technical Paper $110727,2011$.

[6] Barton, J. J., Principles and practice of heating and ventilating, George Newnes Ltd., London, 1964.

[7] Carrier Air Conditioning Company, Handbook of air conditioning system design part 2: Air Distribution, McGraw - Hill, New York, 1972.

[8] Sodiki, J. I., Statistical modeling of head loss components in ventilation ducts, SERSC International Journal of Advanced Science and Technology, vol. 71, 2014, p. $31-40$.

[9] Sodiki, J. I., The head loss ratio in conditioned air distribution: case study of an office block, European Journal of Engineering and Technology, vol. 3, no. 5, 2015, p. 17 - 35. 